\title{
Photovoltaic panel temperature and heat distribution analysis for thermoelectric generator application
}

\begin{abstract}
An experimental study has been conducted to analyze the feasibility of converting the excess heat from PV panels into electrical energy by observing the temperature levels and heat distribution of typical monocrystalline silicon (mono cSi) photovoltaic (PV) panels for photovoltaic-thermoelectric generator (PV-TEG) hybrid application in tropical climate, for example in a Hybrid Agrivoltaic (HAV) Greenhouse System project. It was concluded that the excess heat from the bottom surface of PV panels can be utilize by converting the heat via heat differential to harvest extra electrical energy by applying TEG modules or system. From the experimental results, the highest temperature of the bottom PV panel surface panel recorded at $81.1^{\circ} \mathrm{C}$ during solar noon and expected to reach even higher during hot weather season. The highest power output from the 160 numbers TEG modules in series and parallel arrangement were expected to reach 119 Watt in the afternoon on a normal sunny day. This output is expected to fluctuate over the weather temperature fluctuation throughout the day.
\end{abstract}

Keyword: Photovoltaic (PV); Solar; Thermoelectric (TE); Thermoelectric generator (TEG); Hybrid; Greenhouse system; Agrivotaic system 